\title{
Structure of the Deoxytetranucleotide d-pApTpApT and a Sequence-Dependent Model for Poly (dA-dT)*
}

\author{
M. A. VISWAMITRA, Department of Physics, Indian Institute of \\ Science, Bangalore 560012, India; ZIPPORA SHAKKED, Department \\ of Structural Chemistry, The Weizmann Institute of Science, Rehovot, \\ Israel; and P. G. JONES, G. M. SHELDRICK, S. A. SALISBURY, and \\ OLGA KENNARD, University Chemical Laboratory, Lensfield Road, \\ Cambridge, United Kingdom
}

\begin{abstract}
Synopsis
The $x$-ray structure of the hydrated ammonium salt of the deoxytetranucleotide dpApTpApT was determined by Patterson and direct methods at a resolution of $1 \AA$. The crystal structure contains right-handed double-helical segments formed by complementary Watson-Crick-type hydrogen bonding between the adenine and thymine bases of neighboring molecules. The minihelix contains two base pairs. The chains are antiparallel. The A-T and $\mathrm{T}$-A sequences have different phosphodiester conformations. The deoxyribose-pucker and the sugar-base orientation alternate along the chain depending on the nature of the base ( 3 -endo for purine and $2^{\prime}$-endo for pyrimidine). The extended structure is stabilized by base-base, base-sugar, and hydrogen-bond interactions. The minihelix of two base pairs provides starting coordinates for model-building studies of the dA-dT polymer. A B-DNAtype polymer structure is described, which has sequence-dependent alternations of both the deoxyribose pucker and the phosphate diester bridge conformation. Such sequence-dependent DNA structures, if present locally in regions such as operator sequences, could facilitate sequence-specific interactions. The crystal study also suggests possible geometrical parameters for the replication fork.
\end{abstract}

\section{INTRODUCTION}

Since the discovery of the DNA double helix by Watson and Crick, ${ }^{1} \mathrm{a}$ large body of physicochemical and biological information has accumulated on the structure of the genetic material. Many details of different helical structures of DNA and the influence of environmental factors such as hydration and the presence of metal ions, as well as the dependence of the DNA secondary structure on base composition and sequence, have been obtained mainly from analyses of x-ray fiber-diffraction patterns. ${ }^{2}$

Much of today's thinking is directed at an understanding of how the nucleotide sequences of the double helix are selectively recognized by proteins. This requires a knowledge of the way in which specific base pairs

* Supplementary material on observed and calculated structure factors for d-pApTpApT is available from the authors. 
and base sequences affect the finer details of the DNA structure. Such information cannot be obtained by the methods mentioned above, since the limited data they provide can only be used to visualize an overall averaged structure of the nucleic acid. Geometrical information about the detailed characteristics of the DNA double helix has only recently been obtained from the single-crystal analyses of the tetranucleotide d(pApT. pApT $),{ }^{3}$ the hexanucleotide $\mathrm{d}\left(\mathrm{CpGpCpGpCpG)},{ }^{4}\right.$ and of the tetranucleotide $\mathrm{d}\left(\mathrm{CpGpCpG).5,6} \mathrm{d}(\mathrm{pApTpAp} T)^{3}\right.$ provided, for the first time, geometrical details at atomic resolution on right-handed double-helical fragments of DNA of the B-type. It also suggested a sequence-dependent alternating B-DNA-type structure for poly $(\mathrm{dA}-\mathrm{dT})$. The other oligonucleotides showed left-handed double-helical structures with a zigzag backbone.

A more recent work is concerned with the crystal structure analysis of

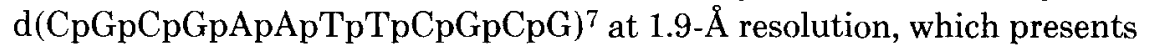
a complete turn of B-DNA.

This paper describes the detailed crystal and molecular structures of the oligodeoxynucleotide $5^{\prime}$-P-adenylyl- $3^{\prime}, 5^{\prime}$-thymidylyl $-3^{\prime}, 5^{\prime}$-adenylyl $-3^{\prime}, 5^{\prime}$ thymidine d(pApTpApT) (Fig. 1) based on an $\mathrm{x}$-ray analysis of its ammonium salt. A double-helical fragment consisting of two base pairs found in the crystal structure was used as a starting point in computer modelbuilding studies to generate a B-DNA-type (dA-dT) polymer having a sequence-dependent sugar-phosphate backbone conformation.

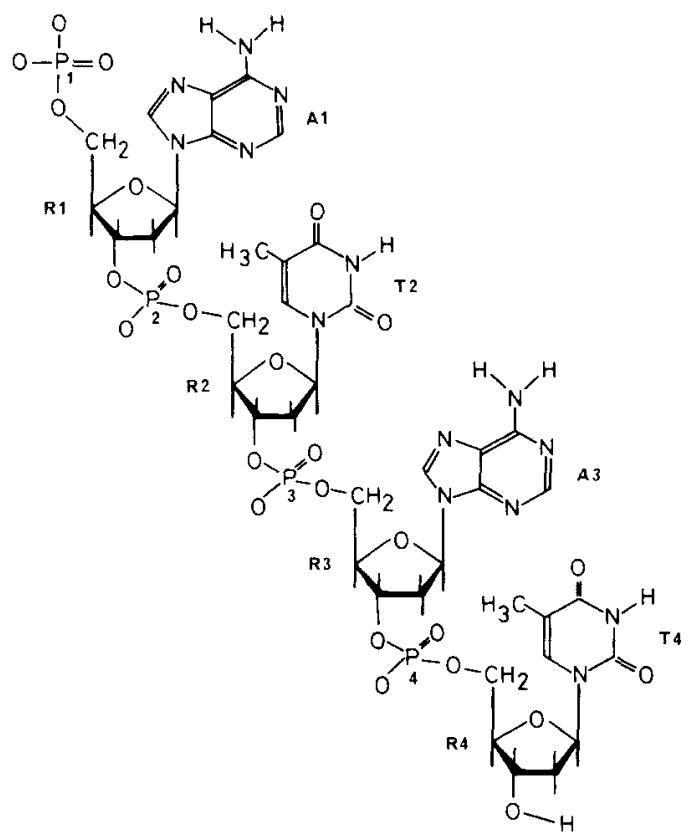

Fig. 1. Chemical formula and residue numbering. 


\section{EXPERIMENTAL}

d-pApTpApT was synthesized by chemically polymerizing the dinucleotide $\mathrm{d}-\mathrm{pAp} \mathrm{T}$ using the diester approach. ${ }^{8}$ The material was purified by ion-exchange chromatography on columns of DEAE cellulose. The ammonium salt of the deoxytetranucleotide was crystallized at $\mathrm{pH} 7.5$ as fine needles on diffusion of acetone into the aqueous solution over a period of about 2 weeks. The crystals were extremely hygroscopic and also readily damaged in the presence of excess acetone. After many attempts an undamaged single crystal was sealed in a capillary tube with mother liquor containing some additional acetone, and this was used for all experimental work.

The crystals were monoclinic with space group $\mathrm{P} 2_{1}$ and cell constants $a=21.121(10), b=21.294(14), c=8.770(4) \AA, \beta=97.84(4)^{\circ}$. The density, measured in a bromoform-xylene gradient column was 1.525 (5) $\mathrm{g}$ $\mathrm{cm}^{-3}$, indicating the presence of two oligomers and 62 species with $M_{r}=$ $18\left(\mathrm{H}_{2} \mathrm{O}\right.$ or $\left.\mathrm{NH}_{4}^{+}\right)$in the unit cell $\left(d_{\text {cal }}=1.52 \mathrm{~g} \mathrm{~cm}^{-3}\right)$. Intensities were measured on a Syntex $\mathrm{P} 2{ }_{1}$ diffractometer by the $\omega / 2 \theta$ scan technique using a high-intensity x-ray tube with monochromatized $\mathrm{MoK}_{\alpha}$ radiation. The resolution was $1.04 \AA\left(2 \theta_{\max }=40^{\circ}\right)$. There were no significant changes in the intensities of two monitor reflections during data collection. Measurements were made at 4528 reciprocal lattice points, giving 2717 independent reflections with $F \geq 1.5 \sigma(F)$. These were used, after the usual corrections, for the solution and refinement of the structure.

d-pApTpApT has a self-complementary base sequence. Theoretically, therefore, it could crystallize as a self-paired duplex of four base pairs. The present structure, however, contained two oligomers related by a two-fold screw axis, which ruled out this possibility and suggested that the actual structure would be significantly different from the usual double helix.

Determination of the structure was not straightforward. Initially we attempted to solve the phase problem by direct methods using both the SHELX and the MULTAN ${ }^{9}$ sets of programs in combination with a wide variety of modifications to the starting phase set. Some of the $E$-maps obtained from these calculations contained covalently linked planar fragments corresponding to adenine. All attempts to derive the structure from phase expansions based on these fragments failed.

As an alternative, skeletal and CPK models of the tetranucleotide were built and positioned in the unit cell with the bases oriented along the (604) plane (which had the highest $E_{\text {obs }}$ value). Information on the packing of RNA double-helical fragments $\mathrm{ApU}^{10}$ and $\mathrm{GpC},{ }^{11,12}$ which crystallize in the same space group, was also used. The model and its orientation were adjusted with due regard to the restrictions imposed by the short $8.8-\AA c$ axis to enable the generation of Watson-Crick-type base pairs by suitable screw rotations and cell translations. From these considerations, a tetranucleotide model consisting of two helical dinucleotide units seemed most favorable. However, the nature of the link between the two units was not clear at that stage. This model served as a guide in locating the posi- 
tions of the $\mathrm{P}$ atoms from a sharpened Patterson map. Phases generated for the two $P$ atoms that could be located most precisely were used as a starting point for tangent expansion following closely the method described by Karle. ${ }^{13}$ An almost complete structure was generated in six cycles. The remaining atoms, as well as the water molecules (some possibly $\mathrm{NH}_{4}^{+}$ions), distributed among 42 sites, were located from difference maps.

The structure was refined by least-squares to a conventional $R=0.153$. The site occupancies of the water molecule/ $\mathrm{NH}_{4}^{+}$ions were included among the variables. It was decided to refine the site occupanies of the solvent molecules rather than their temperature factors, since several water-water contacts were shorter than $2 \AA$, indicating that the water molecules are disordered. Thirty-six $\mathrm{H}$ atoms of the tetranucleotide molecule were fixed at calculated positions. Only $\mathrm{P}$ atoms were allowed anisotropic temperature factors. It was not possible to refine the structure further because of the disordered water, and the limited data set.

A view of the molecule perpendicular to the bases is given in Fig. 2. It shows that the molecule is extended about the middle phosphodiester link.

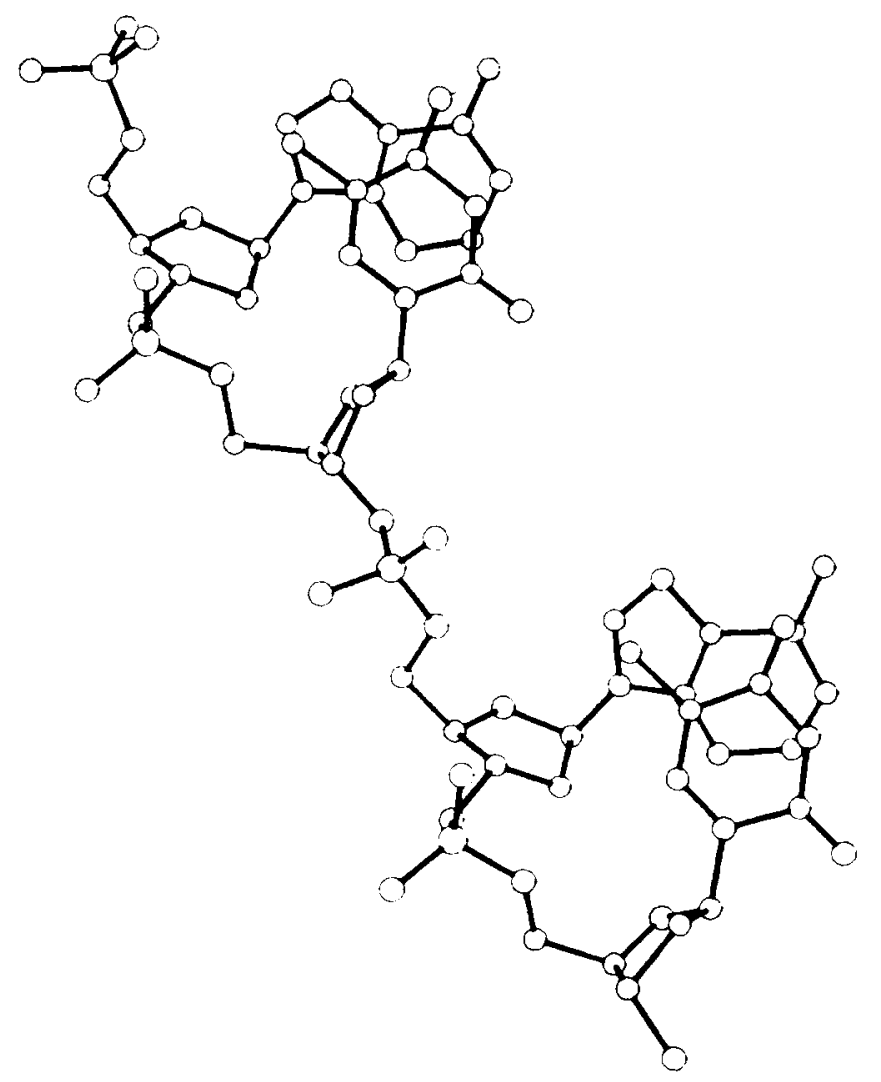

Fig. 2. PLUTO drawing of a single molecule viewed perpendicular to the bases. 
The two halves on either side have very similar conformations. All four nucleotide units have the normal gg conformation about the $\mathrm{C}^{\prime}-\mathrm{C}^{\prime}$ bond.

The final coordinates and thermal parameters of non-hydrogen atoms are listed in Tables I and II; bond lengths and angles are given in Table III.

\section{RESULTS AND DISCUSSION}

\section{Double-Helical Fragment}

The oligomer molecule, as mentioned under Experimental, cannot form a self-complementary double helix of four base pairs in the present structure, which only has a 2 symmetry operator. A segment of a right-handed, antiparallel, two-base-pair double helix is, however, formed with complementary hydrogen bonding between adenine and thymine bases from two molecules related by the $2_{1}$ axis $\left(\mathrm{A} 1 \cdot \mathrm{T} 4^{*}, \mathrm{~T} 2 \cdot \mathrm{A} 3^{*}\right.$; starred bases at $1-x$, $-1 / 2+y,-z)$. The converse pairs $\mathrm{T} 4 \cdot \mathrm{Al}^{*}$ and $\mathrm{A} 3 \cdot \mathrm{T} 2 *$ have the starred bases at $1-x, 1 / 2+y,-z$; thus each molecule contributes to two minihelices, one with each of two other molecules related by a $y$ translation.

Figure 3 gives views of this minihelix perpendicular and parallel to the base pairs. Each base pair is held by hydrogen bonds between the N6 amino group of adenine and the $\mathrm{O} 4$ carbonyl group of thymine (3.04 $\AA$ av.) and between $\mathrm{N} 1$ of adenine and N3 of thymine $(2.82 \AA \mathrm{av}$.) in the classical Watson-Crick geometry, which is seen here for the first time for adeninethymine at atomic resolution in a single-crystal study.

Within each base pair the pyrimidine and purine bases are inclined to each other with a dihedral angle of $13^{\circ}$ between $\mathrm{A} 1$ and $\mathrm{T} 4^{*}$ and $14^{\circ}$ between $\mathrm{T} 2$ and $\mathrm{A} 3^{*}$. A similar propeller twist that increases the overlap between one base and its neighbors is observed in the dodecamer d(CGCGAATTCGCG) ${ }^{7}$ and was proposed by Levitt from energy calculations. ${ }^{14}$ The mean planes through the base pairs $\mathrm{A} 1 \cdot \mathrm{T} 4{ }^{*}$ and $\mathrm{T} 2 \cdot \mathrm{A} 3^{*}$ are nearly parallel to each other $\left(3^{\circ}\right)$, with a vertical separation of $3.34 \AA$. The angular orientation of the base pairs, defined by the angle between vectors $\mathrm{C1}^{\prime} \mathrm{R} 1-\mathrm{C} 1^{\prime} \mathrm{R} 4^{*}$ and $\mathrm{C} 1^{\prime} \mathrm{R} 2-\mathrm{C} 1^{\prime} \mathrm{R} 3^{*}$, is $31^{\circ}$, and the $\mathrm{C} 1^{\prime}-\mathrm{C} 1^{\prime}$ interstrand separation is $10.2 \AA$, close to that found in ApU. ${ }^{10}$ This distance is significantly shorter than the corresponding distance found in the structure of GpC. ${ }^{11,12}$

\section{Sugar Conformation and Sugar-Base Orientation}

A striking feature of the d-pApTpApT molecule, and hence of the minihelix, is the alternation of the sugar conformation between $\mathrm{C} 3$ '-endo and $\mathrm{C} 2$ '-endo pucker, depending on whether the sugar is attached to a purine or pyrimidine base. This is in contrast to the classical interpretations of DNA fiber patterns based on monotonic sugar geometry, $\mathrm{C}^{\prime}$-endo in A-DNA and $\mathrm{C} 2^{\prime}$-endo in B-DNA. ${ }^{2}$ 
TABLE I

Atomic Coordinates and Isotropic Temperature Factors ${ }^{\mathrm{a}}$

\begin{tabular}{|c|c|c|c|c|}
\hline Atom & $10^{4} x / a$ & $10^{4} y / b$ & $10^{4} z / c$ & $10^{3} \mathrm{U}$ \\
\hline P4 & $1399(4)$ & $7400(6)$ & $-815(13)$ & * \\
\hline $\mathrm{O} 1 \mathrm{P} 4$ & $1304(11)$ & $6786(12)$ & $-1700(26)$ & $101(8)$ \\
\hline $\mathrm{O} 2 \mathrm{P} 4$ & $863(13)$ & $7758(13)$ & $-376(30)$ & $127(9)$ \\
\hline $\mathrm{O} 3 \mathrm{P} 4$ & $1868(9)$ & $7331(9)$ & $681(21)$ & $67(6)$ \\
\hline $\mathrm{O5P} 4$ & $1777(9)$ & $7825(10)$ & $-1790(22)$ & $81(6)$ \\
\hline P3 & $1803(4)$ & $5031(4)$ & $1511(10)$ & $*$ \\
\hline O1P3 & $1892(11)$ & $4771(12)$ & $171(29)$ & $111(8)$ \\
\hline $\mathrm{O} 2 \mathrm{P} 3$ & $1140(9)$ & $5165(9)$ & $1799(21)$ & $69(6)$ \\
\hline O3P3 & $2158(7)$ & $4658(8)$ & $2871(17)$ & $47(5)$ \\
\hline O5P3 & $2211(8)$ & $5626(8)$ & $1569(18)$ & $56(5)$ \\
\hline $\mathrm{P} 2$ & $1045(4)$ & $2843(5)$ & $6669(9)$ & * \\
\hline O1P2 & $496(12)$ & $3221(12)$ & $7106(27)$ & $112(8)$ \\
\hline $\mathrm{O} 2 \mathrm{P} 2$ & $887(8)$ & $2262(10)$ & $5805(20)$ & $65(5)$ \\
\hline O3P2 & $1448(9)$ & $2712(9)$ & $8264(21)$ & $70(6)$ \\
\hline $\mathrm{O5P2}$ & $1499(7)$ & $3220(8)$ & $5698(18)$ & $48(5)$ \\
\hline P1 & $1535(5)$ & $322(0)$ & $8781(12)$ & $*$ \\
\hline O1P1 & $1588(13)$ & $87(14)$ & $7263(34)$ & $136(10)$ \\
\hline $\mathrm{O} 2 \mathrm{P} 1$ & $918(18)$ & $242(19)$ & $9266(42)$ & $191(14)$ \\
\hline O3P1 & $2106(24)$ & $-61(26)$ & $9932(60)$ & $298(23)$ \\
\hline O5P1 & $1759(8)$ & $1017(8)$ & $8839(19)$ & $57(5)$ \\
\hline O1R4 & $3001(10)$ & $8382(11)$ & $-2324(23)$ & $85(6)$ \\
\hline C1R4 & $2997(14)$ & $8265(15)$ & $-3942(33)$ & $71(9)$ \\
\hline $\mathrm{C} 2 \mathrm{R} 4$ & $2393(11)$ & $8275(12)$ & $-4791(29)$ & $47(7)$ \\
\hline C3R4 & $2066(17)$ & $8796(18)$ & $-3905(38)$ & $100(11)$ \\
\hline O3R4 & $2229(12)$ & $9451(13)$ & $-4583(30)$ & $122(9)$ \\
\hline C4R4 & $2451(13)$ & $8721(14)$ & $-2300(30)$ & $65(9)$ \\
\hline C5R4 & $1973(16)$ & $8420(16)$ & $-1280(37)$ & $85(10)$ \\
\hline O1R3 & $3179(8)$ & $6565(9)$ & $2746(19)$ & $58(5)$ \\
\hline C1R3 & $3578(11)$ & $6889(12)$ & $1791(27)$ & $43(7)$ \\
\hline C2R3 & $3064(13)$ & $7258(16)$ & $776(35)$ & $80(9)$ \\
\hline C3R3 & $2427(9)$ & $6943(10)$ & $798(23)$ & $26(6)$ \\
\hline C4R3 & $2554(12)$ & $6672(13)$ & $2481(29)$ & $55(7)$ \\
\hline C5R3 & $2156(13)$ & $6100(14)$ & $2852(31)$ & $60(8)$ \\
\hline O1R2 & $2697(8)$ & $3590(8)$ & $5191(18)$ & $53(5)$ \\
\hline C1R2 & $2738(12)$ & $3364(13)$ & $3574(29)$ & $49(8)$ \\
\hline $\mathrm{C} 2 \mathrm{R} 2$ & $2141(16)$ & $3503(18)$ & $2819(41)$ & $97(11)$ \\
\hline C3R2 & $1917(12)$ & $4112(12)$ & $3519(28)$ & $50(7)$ \\
\hline $\mathrm{C} 4 \mathrm{R} 2$ & $2210(11)$ & $4044(12)$ & $5003(27)$ & $43(7)$ \\
\hline C5R2 & $1716(16)$ & $3875(17)$ & $6231(39)$ & $97(11)$ \\
\hline O1R1 & $2708(9)$ & $1846(10)$ & $10383(22)$ & $82(6)$ \\
\hline C1R1 & $3129(12)$ & $2202(13)$ & $9405(27)$ & $47(7)$ \\
\hline C2R1 & $2638(12)$ & $2631(14)$ & $8441(32)$ & $62(8)$ \\
\hline C3R1 & $1984(11)$ & $2316(12)$ & $8473(28)$ & $45(7)$ \\
\hline C4R1 & $2015(10)$ & $2029(11)$ & $9935(25)$ & $36(6)$ \\
\hline C5R1 & $1684(12)$ & $1431(12)$ & $10058(28)$ & $52(8)$ \\
\hline $\mathrm{N} 1 \mathrm{~T} 4$ & $3289(11)$ & $7601(11)$ & $-3820(26)$ & $66(7)$ \\
\hline $\mathrm{C} 2 \mathrm{~T} 4$ & $3908(12)$ & $7505(13)$ & $-4450(29)$ & $50(7)$ \\
\hline $\mathrm{O} 2 \mathrm{~T} 4$ & $4179(8)$ & $7969(9)$ & $-4892(20)$ & $\begin{array}{c}67(5) \\
\text { (continued) }\end{array}$ \\
\hline
\end{tabular}


TABLE I (continued)

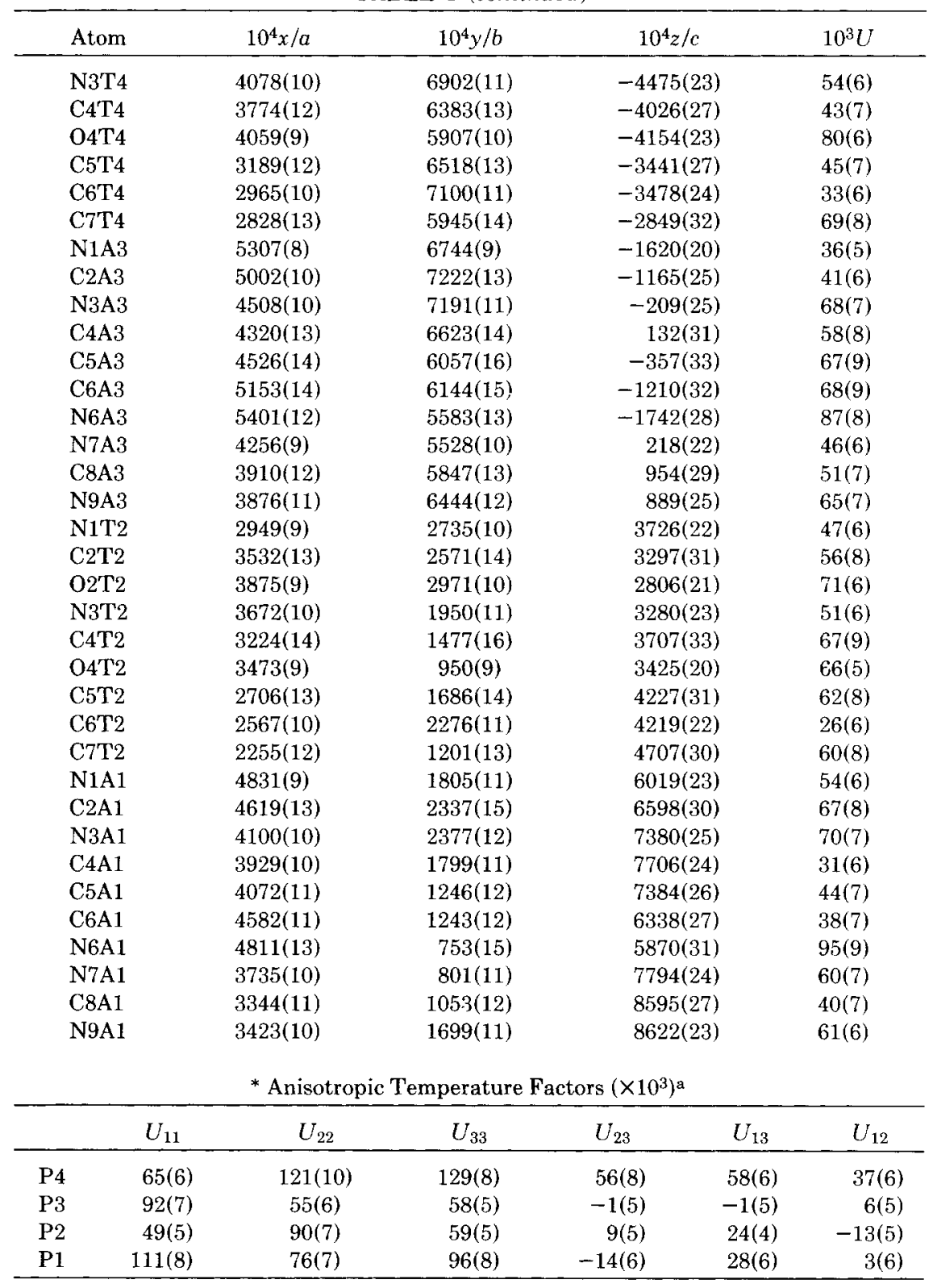

a Temperature factor units are $\AA^{2}$. The temperature factor exponent takes the following form: $-2 \pi^{2}\left(U_{11} h^{2} a *^{2}+\cdots+2 U_{12} h k a^{*} b^{*}\right)$.

The relative orientations of the base and sugar, defined by the glycosidic torsion angles $\chi_{\mathrm{CN}}$, are anti for the four bases. The $\chi_{\mathrm{CN}}$ values are almost at the limits of those found in single-crystal studies ${ }^{15}: \mathrm{O}^{\prime}-\mathrm{Cl}^{\prime}-\mathrm{N} 9-\mathrm{C} 8,5^{\circ}$ (A1) and $-9^{\circ}$ (A3) and $01^{\prime}-\mathrm{C} 1^{\prime}-\mathrm{N} 1-\mathrm{C} 6,69^{\circ}$ (T2) and $75^{\circ}$ (T4). A negative 
TABLE II

Water/Ammonium Ion Coordinates and Site Occupation Factors $(\alpha)^{\text {a }}$

\begin{tabular}{|c|c|c|c|c|}
\hline & $10^{4} x / a$ & $10^{4} y / b$ & $10^{4} z / c$ & $\alpha$ \\
\hline Ow1 & $9082(15)$ & $7143(17)$ & $7297(36)$ & $0.97(4)$ \\
\hline Ow2 & $1230(14)$ & $6902(16)$ & $5039(35)$ & $1.05(4)$ \\
\hline Ow3 & $214(22)$ & $9163(24)$ & $5603(53)$ & $0.65(4)$ \\
\hline Ow4 & $9383(25)$ & $5915(27)$ & $4487(61)$ & $0.58(4)$ \\
\hline Ow5 & $6442(39)$ & $9855(42)$ & $6402(96)$ & $0.37(4)$ \\
\hline Ow6 & $1297(18)$ & $5465(20)$ & $7593(44)$ & $0.77(4)$ \\
\hline Ow7 & $4138(26)$ & $8452(28)$ & $512(64)$ & $0.56(4)$ \\
\hline Ow8 & $643(32)$ & $5150(45)$ & $4799(80)$ & $0.47(5)$ \\
\hline Ow9 & $6949(19)$ & $9217(21)$ & $42(47)$ & $0.76(4)$ \\
\hline Ow10 & $648(38)$ & $5646(53)$ & $4561(91)$ & $0.43(5)$ \\
\hline Ow11 & $6316(24)$ & $9671(26)$ & $4815(59)$ & $0.62(4)$ \\
\hline Ow12 & $5855(42)$ & $4442(47)$ & $2902(102)$ & $0.38(4)$ \\
\hline Ow13 & $824(29)$ & $7890(34)$ & $2827(73)$ & $0.49(4)$ \\
\hline Ow14 & $804(35)$ & $5582(39)$ & $8282(82)$ & $0.41(4)$ \\
\hline Ow15 & $6123(24)$ & $8825(27)$ & $2007(61)$ & $0.69(4)$ \\
\hline Ow16 & $5913(36)$ & $8412(42)$ & $1109(93)$ & $0.42(4)$ \\
\hline Ow17 & $1283(24)$ & $9355(26)$ & $2340(58)$ & $0.61(4)$ \\
\hline Ow18 & $774(43)$ & $7124(49)$ & $2944(104)$ & $0.35(4)$ \\
\hline Ow19 & $58(28)$ & $6405(29)$ & $8145(64)$ & $0.54(4)$ \\
\hline Ow20 & $3572(31)$ & $8728(33)$ & $2383(75)$ & $0.49(4)$ \\
\hline Ow21 & $9419(23)$ & $9089(25)$ & $723(54)$ & $0.64(4)$ \\
\hline Ow22 & $9758(23)$ & $8257(27)$ & $8620(58)$ & $0.61(4)$ \\
\hline Ow23 & $2763(35)$ & $9819(38)$ & $2362(85)$ & $0.44(4)$ \\
\hline Ow24 & $4244(33)$ & $4272(34)$ & $1925(77)$ & $0.49(4)$ \\
\hline Ow25 & $9492(40)$ & $5779(43)$ & $7801(95)$ & $0.37(4)$ \\
\hline Ow26 & $5315(62)$ & $4275(60)$ & $4238(132)$ & $0.29(4)$ \\
\hline Ow27 & $4896(49)$ & $3634(56)$ & $3460(118)$ & $0.31(4)$ \\
\hline Ow28 & $4636(58)$ & $4204(56)$ & $4397(126)$ & $0.30(4)$ \\
\hline Ow29 & $4712(40)$ & $4296(41)$ & $909(92)$ & $0.39(4)$ \\
\hline Ow30 & $210(40)$ & $4525(47)$ & $6458(95)$ & $0.37(4)$ \\
\hline Ow31 & $3622(48)$ & $9544(51)$ & $7293(134)$ & $0.34(5)$ \\
\hline Ow32 & $4762(47)$ & $8879(55)$ & $3363(117)$ & $0.32(5)$ \\
\hline Ow33 & $50(69)$ & $5499(72)$ & $9290(154)$ & $0.23(4)$ \\
\hline Ow34 & $5370(48)$ & $4533(56)$ & $557(123)$ & $0.30(4)$ \\
\hline Ow35 & $3516(26)$ & $9696(29)$ & $4953(64)$ & $0.57(4)$ \\
\hline Ow36 & $6893(42)$ & $9455(47)$ & $1056(103)$ & $0.36(4)$ \\
\hline Ow37 & $9828(54)$ & $7130(61)$ & $658(133)$ & $0.28(4)$ \\
\hline Ow38 & $5005(45)$ & $9244(51)$ & $2473(111)$ & $0.37(4)$ \\
\hline Ow39 & $246(32)$ & $6127(38)$ & $853(83)$ & $0.46(4)$ \\
\hline Ow 40 & $262(52)$ & $6579(59)$ & $2832(134)$ & $0.28(4)$ \\
\hline Ow41 & $9203(45)$ & $4969(49)$ & $4989(113)$ & $0.33(4)$ \\
\hline Ow 42 & $9966(45)$ & $6314(50)$ & $3859(107)$ & $0.37(14)$ \\
\hline
\end{tabular}

a All isotropic temperature factors fixed at $0.15 \AA^{2}$.

value for $\chi$ is particularly uncommon and has been observed in $5^{\prime}$-dCMP $\left(-6^{\circ}\right),{ }^{16}$ where, however, the sugar is $\mathrm{C} 3^{\prime}-$ exo. It should be noted that the sugar conformation in the left-handed $d(C G)_{3}$ structure $^{4}$ also alternates between $\mathrm{C} 2$ '-endo and $\mathrm{C}^{\prime}$-endo for pyrimidines and purines, respectively, whereas the corresponding glycosidic angles exhibit the anti and syn 
TABLE III

Bond Lengths ( $\AA$ ) and Bond Angles (deg)

\begin{tabular}{|c|c|c|c|}
\hline Atoms & Bond Length & Atoms & Bond Length \\
\hline P4-O1P4 & $1.52(3)$ & $\mathrm{P} 4-\mathrm{O} 2 \mathrm{P} 4$ & $1.46(3)$ \\
\hline P4-O3P4 & $1.54(2)$ & $\mathrm{P} 4-\mathrm{O} 5 \mathrm{P} 4$ & $1.54(2)$ \\
\hline P3-O1P3 & $1.34(3)$ & P3-O2P3 & $1.48(2)$ \\
\hline P3-O3P3 & $1.54(2)$ & P3-05P3 & $1.53(2)$ \\
\hline P2-O1P2 & $1.50(3)$ & $\mathrm{P} 2-\mathrm{O} 2 \mathrm{P} 2$ & $1.47(2)$ \\
\hline $\mathrm{P} 2-\mathrm{O} 3 \mathrm{P} 2$ & $1.56(2)$ & $\mathrm{P} 2-05 \mathrm{P} 2$ & $1.58(2)$ \\
\hline P1-O1P1 & $1.44(3)$ & P1-O2P1 & $1.44(4)$ \\
\hline P1-O3P1 & $1.67(5)$ & $\mathrm{P} 1-05 \mathrm{P} 1$ & $1.55(2)$ \\
\hline C1R4-01R4 & $1.44(4)$ & C1R4-C2R4 & $1.39(4)$ \\
\hline C1R4-N1T4 & $1.54(4)$ & C2R4-C3R4 & $1.57(5)$ \\
\hline C3R4-O3R4 & $1.57(5)$ & C3R4-C4R4 & $1.54(4)$ \\
\hline C4R4-O1R4 & $1.37(4)$ & C4R4-C5R4 & $1.57(5)$ \\
\hline C5R4-O5P4 & $1.39(4)$ & C1R3-O1R3 & $1.44(3)$ \\
\hline C1R3-C2R3 & $1.53(4)$ & C1R3-N9A3 & $1.43(4)$ \\
\hline C2R3-C3R3 & $1.51(4)$ & C3R3-O3P4 & $1.43(3)$ \\
\hline C3R3-C4R3 & $1.57(3)$ & C4R3-O1R3 & $1.33(3)$ \\
\hline C4R3-C5R3 & $1.54(4)$ & C5R3-O5P3 & $1.53(3)$ \\
\hline C1R2-O1R2 & $1.51(3)$ & $\mathrm{C} 1 \mathrm{R} 2-\mathrm{C} 2 \mathrm{R} 2$ & $1.37(4)$ \\
\hline C1R2-N1T2 & $1.41(3)$ & $\mathrm{C} 2 \mathrm{R} 2-\mathrm{C} 3 \mathrm{R} 2$ & $1.54(5)$ \\
\hline C3R2-O3P3 & $1.42(3)$ & $\mathrm{C} 3 \mathrm{R} 2-\mathrm{C} 4 \mathrm{R} 2$ & $1.37(3)$ \\
\hline C4R2-O1R2 & $1.41(3)$ & C4R2-C5R2 & $1.64(5)$ \\
\hline C5R2-O5P2 & $1.52(4)$ & C1R1-O1R1 & $1.52(3)$ \\
\hline C1R1-C2R1 & $1.55(4)$ & C1R1-N9A1 & $1.46(4)$ \\
\hline C2R1-C3R1 & $1.54(4)$ & C3R1-O3P2 & $1.40(3)$ \\
\hline C3R1-C4R1 & $1.41(3)$ & C4R1-O1R1 & $1.51(3)$ \\
\hline C4R1-C5R1 & $1.46(4)$ & C5R1-O5P1 & $1.41(3)$ \\
\hline C2T4-N1T4 & $1.50(4)$ & $\mathrm{C} 2 \mathrm{~T} 4-\mathrm{O} 2 \mathrm{~T} 4$ & $1.23(3)$ \\
\hline C2T4-N3T4 & $1.34(4)$ & C4'T4-N3T4 & $1.36(4)$ \\
\hline C4T4-04T4 & $1.19(3)$ & $\mathrm{C} 4 \mathrm{~T} 4-\mathrm{C} 5 \mathrm{~T} 4$ & $1.43(4)$ \\
\hline C5T4-C6T4 & $1.33(4)$ & C5T4-C7T4 & $1.56(4)$ \\
\hline C6T4-N1T4 & $1.56(4)$ & C2A3-N1A3 & $1.30(3)$ \\
\hline C2A3-N3A3 & $1.43(3)$ & C4A3-N3A3 & $1.32(4)$ \\
\hline C4A3-C5A3 & $1.37(4)$ & C4A3-N9A3 & $1.28(4)$ \\
\hline C5A3-C6A3 & $1.62(4)$ & C5A3-N7A3 & $1.39(4)$ \\
\hline C6A3-N7A3 & $1.38(4)$ & C6A3-N6A3 & $1.41(4)$ \\
\hline C8A3-N7A3 & $1.24(4)$ & C8A3-N9A3 & $1.27(4)$ \\
\hline C2T2-N1T2 & $1.38(4)$ & $\mathrm{C} 2 \mathrm{~T} 2-\mathrm{O} 2 \mathrm{~T} 2$ & $1.23(4)$ \\
\hline C2T2-N3T2 & $1.36(4)$ & C4T2-N3T2 & $1.47(4)$ \\
\hline $\mathrm{C} 4 \mathrm{~T} 2-\mathrm{O} 4 \mathrm{~T} 2$ & $1.28(4)$ & $\mathrm{C} 4 \mathrm{~T} 2-\mathrm{C} 5 \mathrm{~T} 2$ & $1.32(4)$ \\
\hline $\mathrm{C} 5 \mathrm{~T} 2-\mathrm{C} 6 \mathrm{~T} 2$ & $1.29(4)$ & C5T2-C7T2 & $1.50(4)$ \\
\hline C6T2-N1T2 & $1.37(3)$ & C2A1-N1A1 & $1.34(4)$ \\
\hline C2A1-N3A1 & $1.37(4)$ & C4A1-N3A1 & $1.32(4)$ \\
\hline C4A1-C5A1 & $1.26(4)$ & C4A1-N9A1 & $1.44(3)$ \\
\hline C5A1-C6A1 & $1.51(4)$ & C5A1-N7A1 & $1.27(3)$ \\
\hline C6A1-N1A1 & $1.35(3)$ & C6A1-N6A1 & $1.24(4)$ \\
\hline C8A1-N7A1 & $1.27(3)$ & C8A1-N9A1 & $\begin{array}{c}1.38(3) \\
\text { (continued) }\end{array}$ \\
\hline
\end{tabular}


TABLE III (continued)

\begin{tabular}{|c|c|c|c|}
\hline Atoms & Bond Angle & Atoms & Bond Angle \\
\hline $\mathrm{O} 1 \mathrm{P} 4-\mathrm{P} 4-02 \mathrm{P} 4$ & $122(2)$ & O1P4-P4-O3P4 & $112(1)$ \\
\hline $\mathrm{O} 1 \mathrm{P} 4-\mathrm{P} 4-05 \mathrm{P} 4$ & $105(1)$ & O2P4-P4-O3P4 & $105(1)$ \\
\hline $\mathrm{O} 2 \mathrm{P} 4-\mathrm{P} 4-05 \mathrm{P} 4$ & $109(2)$ & O3P4-P4-05P4 & $102(1)$ \\
\hline O1P3-P3-O2P3 & $119(1)$ & O1P3-P3-O3P3 & $111(1)$ \\
\hline O1P3-P3-05P3 & $103(1)$ & O2P3-P3-O3P3 & $110(1)$ \\
\hline О2Р3-P3-O5P3 & $112(1)$ & O5P3-P3-O3P3 & $101(1)$ \\
\hline $\mathrm{O} 1 \mathrm{P} 2-\mathrm{P} 2-\mathrm{O} 2 \mathrm{P} 2$ & $117(1)$ & $\mathrm{O} 1 \mathrm{P} 2-\mathrm{P} 2-\mathrm{O} 3 \mathrm{P} 2$ & $102(1)$ \\
\hline $\mathrm{O} 1 \mathrm{P} 2-\mathrm{P} 2-\mathrm{O} 5 \mathrm{P} 2$ & $114(1)$ & $\mathrm{O} 2 \mathrm{P} 2-\mathrm{P} 2-\mathrm{O} 3 \mathrm{P} 2$ & $112(1)$ \\
\hline $\mathrm{O} 2 \mathrm{P} 2-\mathrm{P} 2-\mathrm{O} 5 \mathrm{P} 2$ & $105(1)$ & $\mathrm{O} 3 \mathrm{P} 2-\mathrm{P} 2-\mathrm{O} 5 \mathrm{P} 2$ & $106(1)$ \\
\hline O1P1-P1-02P1 & $115(2)$ & O1P1-P1-03P1 & $104(2)$ \\
\hline O1P1-P1-O5P1 & $107(2)$ & O2P1-P1-O3P1 & $112(3)$ \\
\hline O2P1-P1-O5P1 & $113(2)$ & 03P1-P1-05P1 & $105(2)$ \\
\hline P4-O3P4-C3R3 & $123(2)$ & P4-05P4-C5R4 & $121(2)$ \\
\hline P3-O3P3-C3R2 & $125(1)$ & P3-05P3-C5R3 & $118(2)$ \\
\hline P2-O3P2-C3R1 & $124(2)$ & P2-O5P2-C5R2 & $119(2)$ \\
\hline P1-O5P1-C5R1 & $124(2)$ & C1R4-O1R4-C4R4 & $102(2)$ \\
\hline O1R4-C1R4-C2R4 & $114(3)$ & O1R4-C1R4-N1T4 & $98(2)$ \\
\hline C2R4-C1R4-N1T4 & $112(2)$ & C1R4-C2R4-C3R4 & $100(2)$ \\
\hline C2R4-C3R4-O3R4 & $108(3)$ & C2R4-C3R4-C4R4 & $100(3)$ \\
\hline O3R4-C3R4-C4R4 & $109(3)$ & O1R4-C4R4-C3R4 & $113(3)$ \\
\hline O1R4-C4R4-C5R4 & $114(2)$ & C3R4-C4R4-C5R4 & $105(2)$ \\
\hline O5P4-C5R4-C4R4 & $112(3)$ & C1R3-O1R3-C4R3 & $118(2)$ \\
\hline O1R3-C1R3-C2R3 & $99(2)$ & O1R3-C1R3-N9A3 & $110(2)$ \\
\hline C2R3-C1R3-N9A3 & $110(2)$ & C1R3-C2R3-C3R3 & $109(2)$ \\
\hline $\mathrm{O} 3 \mathrm{P} 4-\mathrm{C} 3 \mathrm{R} 3-\mathrm{C} 2 \mathrm{R} 3$ & $118(2)$ & O3P4-C3R3-C4R3 & $108(2)$ \\
\hline C2R3-C3R3-C4R3 & $98(2)$ & O1R3-C4R3-C3R3 & $105(2)$ \\
\hline O1R3-C4R3-C5R3 & $113(2)$ & C3R3-C4R3-C5R3 & $117(2)$ \\
\hline O5P3-C5R3-C4R3 & $106(2)$ & C1R2-O1R2-C4R2 & $104(2)$ \\
\hline $\mathrm{O} 1 \mathrm{R} 2-\mathrm{C} 1 \mathrm{R} 2-\mathrm{C} 2 \mathrm{R} 2$ & $103(2)$ & O1R2-C1R2-N1T2 & $106(2)$ \\
\hline C2R2-C1R2-N1T2 & $121(3)$ & C1R2-C2R2-C3R2 & $108(3)$ \\
\hline O3P3-C3R2-C2R2 & $113(2)$ & O3P3-C3R2-C4R2 & $109(2)$ \\
\hline C2R2-C3R2-C4R2 & $100(2)$ & O1R2-C4R2-C3R2 & $114(2)$ \\
\hline O1R2-C4R2-C5R2 & $107(2)$ & C3R2-C4R2-C5R2 & $114(2)$ \\
\hline $\mathrm{O} 5 \mathrm{P} 2-\mathrm{C} 5 \mathrm{R} 2-\mathrm{C} 4 \mathrm{R} 2$ & $101(2)$ & C1R1-O1R1-C4R1 & $110(2)$ \\
\hline O1R1-C1R1-C2R1 & $102(2)$ & O1R1-C1R1-N9A1 & $103(2)$ \\
\hline C2R1-C1R1-N9A1 & $118(2)$ & C1R1-C2R1-C3R1 & $106(2)$ \\
\hline $\mathrm{O} 3 \mathrm{P} 2-\mathrm{C} 3 \mathrm{R} 1-\mathrm{C} 2 \mathrm{R} 1$ & $116(2)$ & O3P2-C3R1-C4R1 & $108(2)$ \\
\hline C2R1-C3R1-C4R1 & $106(2)$ & O1R1-C4R1-C3R1 & $106(2)$ \\
\hline O1R1-C4R1-C5R1 & $102(2)$ & C3R1-C4R1-C5R1 & $119(2)$ \\
\hline O5P1-C5R1-C4R1 & $113(2)$ & C1R4-N1T4-C2T4 & $117(2)$ \\
\hline C1R4-N1T4-C6T4 & $123(2)$ & C2T4-N1T4-C6T4 & $118(2)$ \\
\hline N1T4-C2T4-O2T4 & $118(2)$ & N1T4-C2T4-N3T4 & $113(2)$ \\
\hline O2T4-C2T4-N3T4 & $129(3)$ & C2T4-N3T4-C4T4 & $130(2)$ \\
\hline N3T4-C4T4-O4T4 & $114(2)$ & N3T4-C4'T4-C5T4 & $114(2)$ \\
\hline O4T4-C4T4-C5T4 & $133(3)$ & C4T4-C5T4-C6T4 & $120(2)$ \\
\hline C4T4-C5T4-C7T4 & $117(2)$ & C6T4-C5T4-C7T4 & $123(2)$ \\
\hline N1T4-C6T4-C5T4 & $124(2)$ & C2A3-N1A3-C6A3 & $120(2)$ \\
\hline N1A3-C2A3-N3A3 & $125(2)$ & C2A3-N3A3-C4A3 & $116(2)$ \\
\hline N3A3-C4A3-C5A3 & $128(3)$ & N3A3-C4A3-N9A3 & $131(3)$ \\
\hline C5A3-C4A3-N9A3 & $101(3)$ & С4A3-С5A3-С6A3 & $\begin{array}{c}111(3) \\
\text { (continued) }\end{array}$ \\
\hline
\end{tabular}


TABLE III (continued)

\begin{tabular}{cccc}
\hline Atoms & Bond Angle & Atoms & Bond Angle \\
\hline C4A3-C5A3-N7A3 & $116(3)$ & C6A3-C5A3-N7A3 & $132(3)$ \\
N1A3-C6A3-C5A3 & $118(3)$ & N1A3-C6A3-N6A3 & $126(3)$ \\
C5A3-C6A3-N6A3 & $115(3)$ & C5A3-N7A3-C8A3 & $93(2)$ \\
N7A3-C8A3-N9A3 & $124(3)$ & C1R3-N9A3-C4A3 & $120(3)$ \\
C1R3-N9A3-C8A3 & $131(2)$ & C4A3-N9A3-C8A3 & $106(3)$ \\
C1R2-N1T2-C2T2 & $120(2)$ & C1R2-N1T2-C6T2 & $121(2)$ \\
C2T2-N1T2-C6T2 & $119(2)$ & N1T2-C2T2-O2T2 & $121(3)$ \\
N1T2-C2T2-N3T2 & $117(2)$ & O2T2-C2T2-N3T2 & $122(3)$ \\
C2T2-N3T2-C4T2 & $121(2)$ & N3T2-C4T2-O4T2 & $105(2)$ \\
N3T2-C4T2-C5T2 & $117(3)$ & O4T2-C4T2-C5T2 & $138(3)$ \\
C4T2-C5T2-C6T2 & $122(3)$ & C4T2-C5T2-C7T2 & $117(3)$ \\
C6T2-C5T2-C7T2 & $121(3)$ & N1T2-C6T2-C5T2 & $124(2)$ \\
C2A1-N1A1-C6A1 & $121(2)$ & N1A1-C2A1-N3A1 & $125(3)$ \\
C2A1-N3A1-C4A1 & $108(2)$ & N3A1-C4A1-C5A1 & $138(3)$ \\
N3A1-C4A1-N9A1 & $120(2)$ & C5A1-C4A1-N9A1 & $102(2)$ \\
C4A1-C5A1-C6A1 & $111(2)$ & C4A1-C5A1-N7A1 & $118(2)$ \\
C6A1-C5A1-N7A1 & $130(2)$ & N1A1-C6A1-C5A1 & $117(2)$ \\
N1A1-C6A1-N6A1 & $120(3)$ & C5A1-N6A1-N6A1 & $123(3)$ \\
C5A1-N7A1-C8A1 & $106(2)$ & N7A1-C8A1-N9A1 & $110(2)$ \\
C1R1-N9A1-C4A1 & $124(2)$ & C1R1-N9A1-C8A1 & $133(2)$ \\
C4A1-N9A1-C8A1 & $104(2)$ & & \\
\hline
\end{tabular}

conformations. However, with the same glycosidic orientations, the sugar conformation in the $\mathrm{d}(\mathrm{CG})_{2}$ structure ${ }^{5,6}$ alternates between $\mathrm{C} 2^{\prime}$-endo and $\mathrm{C} 1$ '-exo for deoxycytidine and deoxyguanosine, respectively.

All these findings suggest that it may be necessary to take into account irregular or periodic variations in sugar-base orientation and sugar pucker parameters, at least over local regions of double-helical structures, depending on the nature of the nucleotide sequence.

\section{The Sugar-Phosphate Backbone}

Figure 4 shows schematically the conformation of the sugar-phosphate backbone. The value of the torsional angle $\psi$ about the $\mathrm{C} 3^{\prime}-\mathrm{C} 4^{\prime}$ bond depends on the sugar conformation and thus alternates along the backbone. The torsion angles $\omega$ and $\omega^{\prime}$ about the P-O5' and P-O3' ester bonds in the $\mathrm{d}(\mathrm{A}-\mathrm{T})$ fragments are close to those allowed for right-handed double-helical polynucleotides. The conformation of the backbone within the two halves of the molecule is remarkably similar. The phosphodiester linkage between $\mathrm{R} 2$ and $\mathrm{R} 3$, however, is very different, with a trans-gauche $\left(\mathrm{tg}^{-}\right)$conformation about the $\mathrm{P} 3-\mathrm{O}^{\prime}$ and $\mathrm{P} 3-\mathrm{O}^{\prime}$ bonds. This conformation gives rise to an extended backbone, similar to that observed in the crystal structures $\mathrm{UpA}^{17,18}$ and pTpT. ${ }^{19}$ In the present structure, however, the $\psi$ values of $\mathrm{R} 2$ and R3 are different, since they relate to the sugar conformation. 

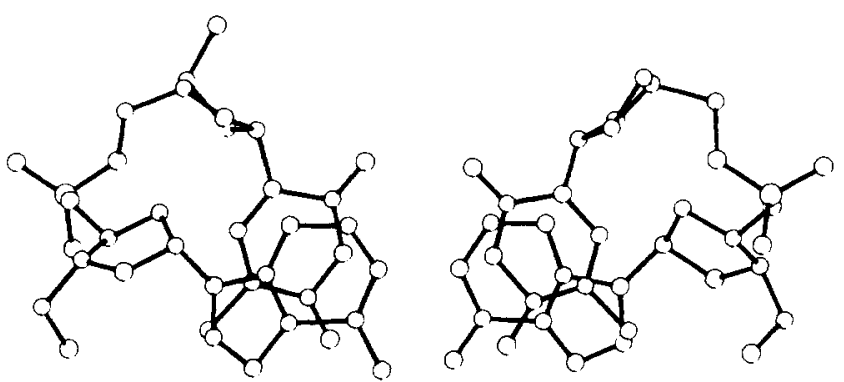

(a)
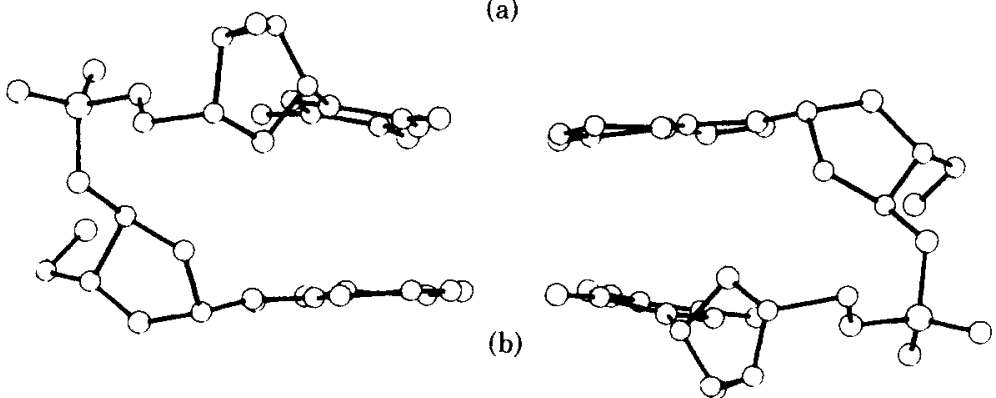

Fig. 3. (a) The minihelix formed by two molecules related by a $2_{1}$ axis, viewed perpendicular to the bases. (b) The minihelix viewed parallel to the bases.

The dimensions of the phosphate group are similar to those observed in the crystal structures of nucleotides containing the phosphodiester linkage. The free P-O bonds are short and the valence angles large (1.45 $\AA$ av., $\mathrm{O} 1-\mathrm{P}-\mathrm{O} 2=118^{\circ}$ av. $)$ compared with the ester bonds and angles $(1.54 \AA$ av., $\mathrm{O} 3-\mathrm{P}-\mathrm{O} 5=104^{\circ} \mathrm{av}$.). The valence angles at $\mathrm{O} 3^{\prime}$ and $\mathrm{O5^{ \prime }}$ are close to $120^{\circ}$. It should, however, be noted that the high thermal parameters of some phosphate oxygens may make some phosphate dimensions imprecise.

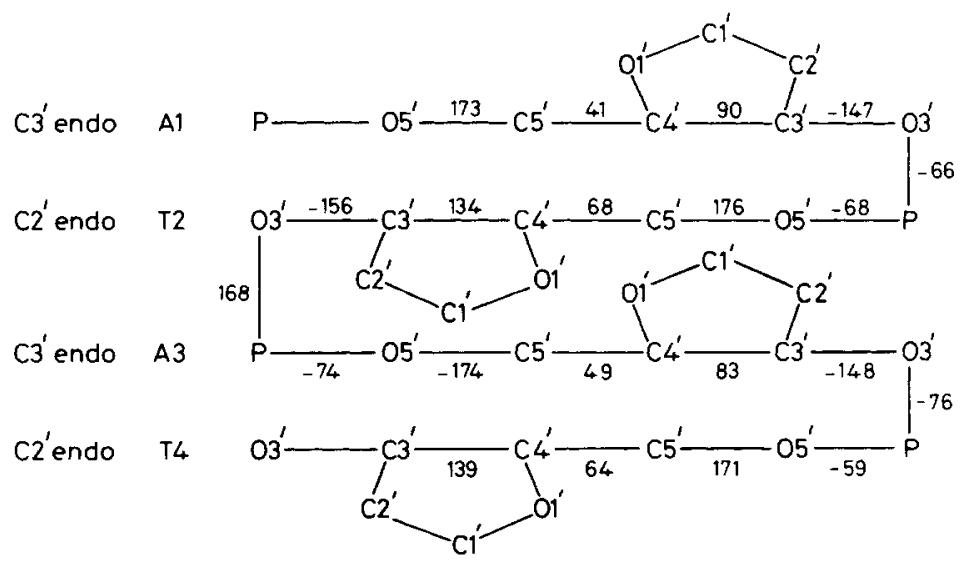

Fig. 4. Schematic view of the sugar-phosphate backbone. 


\section{Base Stacking and Extended Crystal Structure}

Base stacking contributes an important stabilizing force in polynucleotide structure. In d-pApTpApT the conformation of each molecule is stabilized by the considerable overlap between the adenine and thymine bases in the two halves of the molecule and also between the adenines $\mathrm{A} 3$ and $\mathrm{A} 1^{*}$ of two molecules related by a $2_{1}$ axis. The average vertical distance between these bases is $3.5 \AA$. The extended structure is further stabilized by intermolecular base-sugar interactions, similar to those found in UpA. ${ }^{17,18}$ Intramolecular base-sugar stacking was observed in $\mathrm{d}(\mathrm{CG})_{3}{ }^{4}$ and $\mathrm{d}(\mathrm{CG})_{2}$ structures. ${ }^{4-6}$ The stacking arrangements formed in the present structure are shown in the stereo views, Fig. $5(\mathrm{a}, \mathrm{b})$. To place the degree of overlap on a semiquantitative basis, we used potential-energy calculations, ${ }^{20}$ which sum the interaction energy between selected atomic groupings. We find that all three interactions $\mathrm{A} 1: \mathrm{T} 2, \mathrm{~A} 3: \mathrm{T} 4$, and $\mathrm{A} 3: \mathrm{A} 1 *$ are $-5.2 \mathrm{kcal} / \mathrm{mol}$.
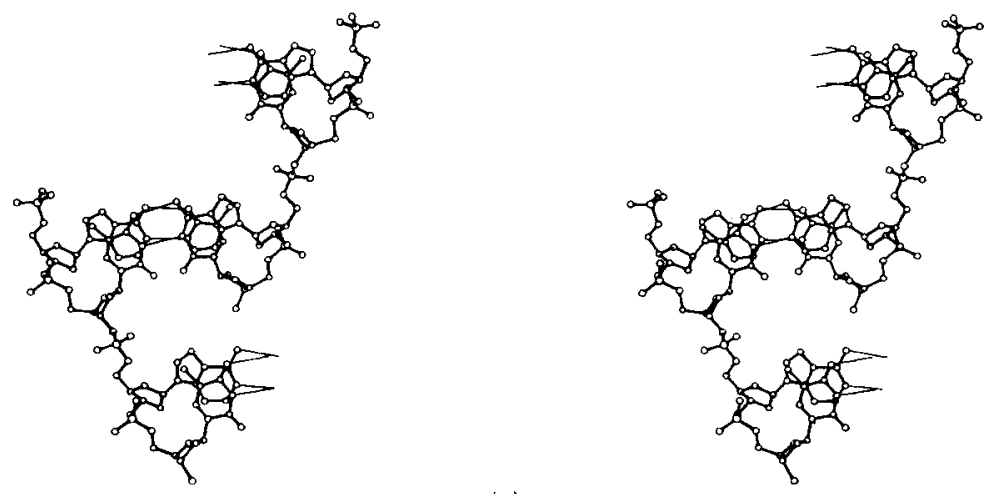

(a)
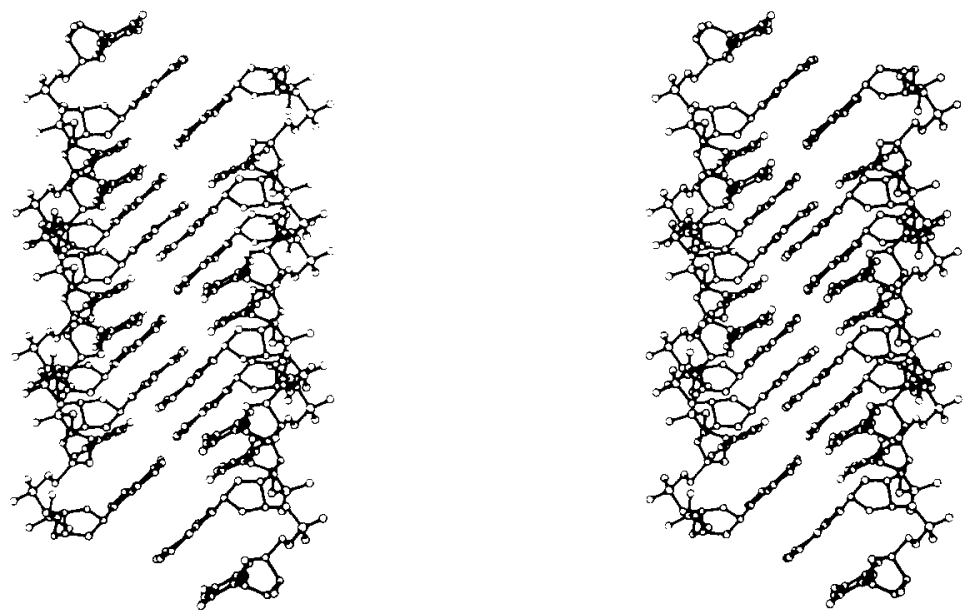

(b)

Fig. 5. (a) Stereodiagram of the two molecules related by the $2_{1}$ screw axis. (b) Stereodiagram of the extended crystal structure showing base-base and base-sugar interactions. 
The interaction energy between thymine and ribose, related by a $c$ translation, is $-3.9 \mathrm{kcal} / \mathrm{mol}$ between $\mathrm{T} 2$ and $\mathrm{R} 1 *$ and $-3.4 \mathrm{kcal} / \mathrm{mol}$ between $\mathrm{T} 4$ and $\mathrm{R}^{*}$ (starred sugars at $x, y,-1+z$ ). These figures are only approximate, but they do indicate the relative contributions of the various interactions.

In addition to these stacking forces, the molecules related by the $b$ translation are held head to tail by a hydrogen bond $2.62 \AA$ long between the $\mathrm{O}^{\prime}$ ' hydroxyl group of one molecule at $x, y, z$ and the free $5^{\prime}$-terminal phosphate oxygen atom O1P1 of a molecule at $x, 1+y, 1+z$.

The extended crystal structure is stabilized by the water molecules and the $\mathrm{NH}_{4}^{+}$ions. Hydrogen-bond distances of the first hydration shell are given in Table IV. All the free phosphate oxygens are coordinated by water $/ \mathrm{NH}_{4}^{+}$ions, and so are the two oxygen atoms of the thymine and $\mathrm{N} 3$, $\mathrm{N} 6$, and $\mathrm{N} 7$ of the adenine bases. In addition, the $\mathrm{O1}^{\prime}$ atoms of the deoxyribose rings of the thymines are coordinated to water molecules Ow11 $(3.1 \AA)$ and Ow31 (2.8 $\AA)$. There is also a network of hydrogen bonds between the water molecules forming a partially ordered structure that extends in infinite columns along the $c$ axis parallel to the water-free columns of stacked base pairs as illustrated in Fig. 6. This structured water provides a major stabilizing force maintaining the d-pApTpApT molecule in the extended crystal structure and accounts for the extreme instability of the crystals in the absence of the mother liquor of precise water content.

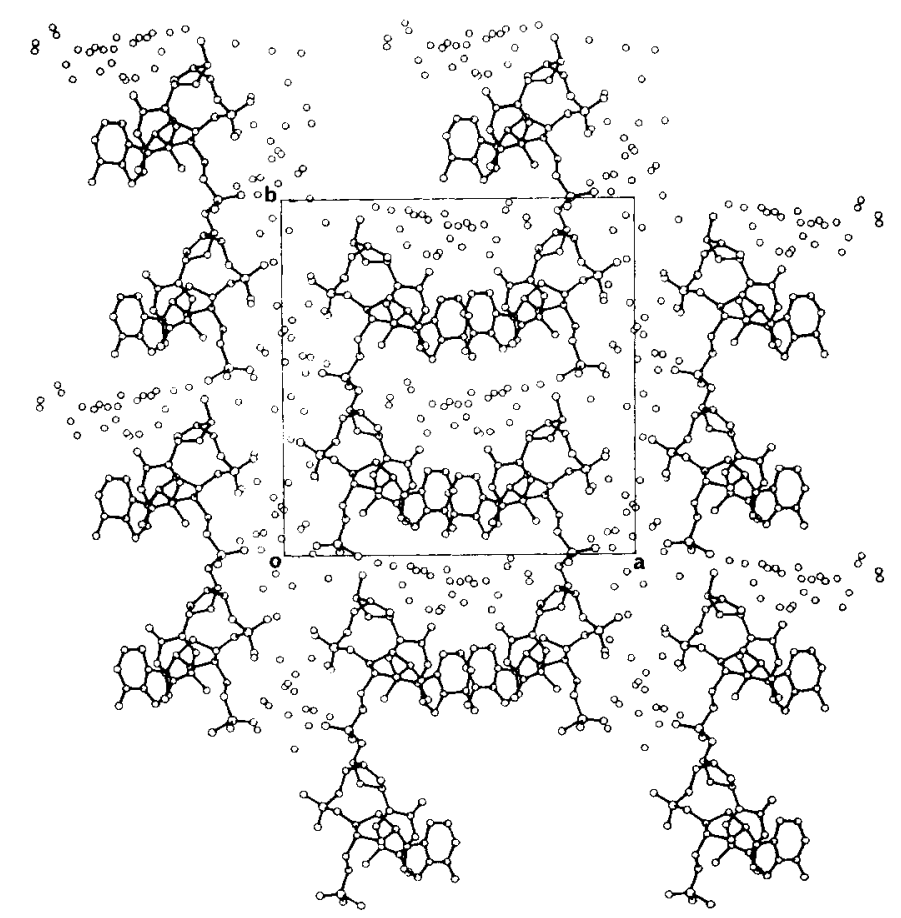

Fig. 6. Packing diagram of the extended crystal structure projected down the $c$ axis. Water oxygens and $\mathrm{N}$ atoms of the $\mathrm{NH}_{4}^{+}$groups are shown as unconnected circles. 
TABLE IV

Hydrogen-Bond Contacts of the First Hydration Shella

\begin{tabular}{|c|c|c|c|c|c|}
\hline & \multirow{2}{*}{$\begin{array}{c}\text { Distance } \\
(\AA)\end{array}$} & \multirow{2}{*}{$\begin{array}{c}\text { Symmetry } \\
\text { Element }\end{array}$} & \multicolumn{3}{|c|}{ Translation Along } \\
\hline & & & $a$ & $b$ & $c$ \\
\hline O1P4... Ow2 & 2.85 & 1 & 0 & 0 & -1 \\
\hline O1P4. . Ow6 & 2.88 & 1 & 0 & 0 & -1 \\
\hline O1P4... Ow14 & 2.77 & 1 & 0 & 0 & -1 \\
\hline O1P4... Ow19 & 2.74 & 1 & 0 & 0 & -1 \\
\hline O2P4... Ow13 & 2.84 & 1 & -1 & 0 & 0 \\
\hline O2P4... Ow22 & 2.61 & 1 & -1 & 0 & -1 \\
\hline O2P4... Ow37 & 2.82 & 1 & -1 & 0 & 0 \\
\hline O1P3...Ow6 & 2.85 & 1 & 0 & 0 & -1 \\
\hline O1P3... Ow9 & 2.75 & 2 & 1 & -1 & 0 \\
\hline O1P3... Ow21 & 3.13 & 2 & 1 & -1 & 0 \\
\hline O1P3... Ow36 & 2.99 & 2 & 1 & -1 & 0 \\
\hline O2P3... Ow8 & 2.96 & 1 & 0 & 0 & 0 \\
\hline O2P3...Ow10 & 2.95 & 1 & 0 & 0 & 0 \\
\hline O2P3... Ow33 & 3.04 & 1 & -1 & 0 & -1 \\
\hline O2P3... Ow39 & 2.83 & 1 & -1 & 0 & 0 \\
\hline O3P3... Ow5 & 2.97 & 2 & 1 & -1 & 1 \\
\hline O1P2... Ow13 & 2.88 & 2 & 1 & -1 & 1 \\
\hline O1P2... Ow21 & 2.64 & 2 & 1 & -1 & 1 \\
\hline O1P2... Ow30 & 2.88 & 1 & 0 & 0 & 0 \\
\hline O2P2... Ow1 & 2.74 & 2 & 1 & -1 & 1 \\
\hline O2P2 . . Ow 4 & 2.93 & 2 & 1 & -1 & 1 \\
\hline $\mathrm{O} 2 \mathrm{P} 2 \ldots \mathrm{OW} 42$ & 2.75 & 2 & 1 & -1 & 1 \\
\hline O1P1... Ow4 & 2.97 & 2 & 1 & -1 & 1 \\
\hline O1P1...Ow41 & 2.42 & 2 & 1 & -1 & 1 \\
\hline O2P1… Ow25 & 3.05 & 2 & 1 & -1 & 2 \\
\hline O2P1… Ow33 & 2.61 & 2 & 1 & -1 & 2 \\
\hline O2P1… Ow39 & 3.09 & 2 & 1 & -1 & 1 \\
\hline O3P1... Ow23 & 2.39 & 1 & 0 & -1 & 1 \\
\hline O1R4...OW31 & 2.84 & 2 & 1 & 0 & 0 \\
\hline O3R4 ‥ Ow17 & 3.14 & 1 & 0 & 0 & -1 \\
\hline O3R4...Ow35 & 2.85 & 1 & 0 & 0 & -1 \\
\hline O1R2... Ow11 & 3.11 & 2 & 1 & -1 & 1 \\
\hline O2T4...Ow20 & 3.02 & 1 & 0 & 0 & -1 \\
\hline O2T4. . Ow26 & 3.01 & 2 & 1 & 0 & 0 \\
\hline O2T4...Ow27 & 2.60 & 2 & 1 & 0 & 0 \\
\hline O2T4. . Ow32 & 2.85 & 1 & 0 & 0 & -1 \\
\hline O4T4...Ow5 & 3.08 & 2 & 1 & -1 & 0 \\
\hline O4T4. O Ow11 & 2.79 & 2 & 1 & -1 & 0 \\
\hline N3A3...Ow7 & 2.89 & 1 & 0 & 0 & 0 \\
\hline N6A3. O Ow34 & 2.98 & 1 & 0 & 0 & 0 \\
\hline N6A3... Ow38 & 3.02 & 2 & 1 & -1 & 0 \\
\hline N7A3 - Ow24 & 3.07 & 1 & 0 & 0 & 0 \\
\hline N7A3 . Ow29 & 2.83 & 1 & 0 & 0 & 0 \\
\hline N7A3 . Ow34 & 3.12 & 1 & 0 & 0 & 0 \\
\hline O2T $2 \cdots$ Ow 24 & 3.01 & 1 & 0 & 0 & 0 \\
\hline $\mathrm{O} 2 \mathrm{~T} 2 \ldots$ Ow 27 & 2.58 & 1 & 0 & 0 & 0 \\
\hline $\mathrm{O} 4 \mathrm{~T} 2 \cdots \mathrm{Ow} 23$ & 2.92 & 1 & 0 & -1 & 0 \\
\hline O4T2...Ow35 & 2.98 & 1 & 0 & -1 & 0 \\
\hline N3A1...Ow16 & 2.57 & 2 & 1 & -1 & 1 \\
\hline N7A1 . O Ow12 & 3.11 & 2 & 1 & -1 & 1 \\
\hline N7A1...Ow31 & 2.72 & 2 & 1 & -1 & 1 \\
\hline
\end{tabular}

a 'The second atom in each pair is related to the first by the corresponding symmetry and translation: (1) $x, y, z ;(2)-x,(1 / 2)+y,-z$. The average esd is $0.08 \AA$. 


\section{Poly(dA-dT) Model}

The minihelix (A1-T2).(A3*-T4*) found in the crystal structure provides a starting point for generating various models for the (dA-dT) copolymer. The coordinates of the minihelix are given in Table V. We have generated a right-handed double helix with the helix axis passing through the base pairs. Models built by hand and using computer programs ${ }^{21}$ showed that no helical structure could be generated from the crystal structure by helical rotation and translation only; it was necessary to make a major change in the conformation of the phosphodiester bridge between T2 and A3 ( $\omega^{\prime}$, the torsion angle about the $\mathrm{O} 3^{\prime}-\mathrm{P}$ bond, being changed from $168^{\circ}$ to $-125^{\circ}$; and $\phi$, the torsion angle about the $\mathrm{C} 3^{\prime}-\mathrm{O} 3^{\prime}$ bond, from $-156^{\circ}$ to $-175^{\circ}$ ). The resultant polymer is of the B-DNA type, with approximately 10 base pairs per turn and a pitch of $33 \AA$ (Fig. 7). It differs from the classical DNA structure in having an alternating, sequence-dependent conformation and hence a dinucleotide repeat unit. In the present model, the A-T overlap is larger, whereas the T-A overlap is smaller than that of B-DNA. ${ }^{22}$ Such a poor T-A overlap may account for the observed break of the helical direction from $T$ to $A$ in the crystal structure. The intermolecular adenine-adenine and ribose-thymine interactions (Fig. 5) that are allowed by this break more than compensate for the loss of intramolecular T-A stacking energy. The model embodies the alternating sugar conformation found in the crystal structure and also shows a difference in phosphate diester conformation between $\mathrm{A}-\mathrm{T}$ and $\mathrm{T}$-A sequences $\left(\omega^{\prime} \simeq-70^{\circ}\right.$ between $\mathrm{A}$ and $\mathrm{T}, \simeq-125^{\circ}$ between $\mathrm{T}$ and $\left.\mathrm{A}\right)$. The helical turn per base pair is also different for the two sequences: $31^{\circ}$ between $\mathrm{A}$ and $\mathrm{T}$ and $41^{\circ}$ between $\mathrm{T}$ and A.

Such a sequence-dependent variation in the local conformation of the deoxyribose-phosphodiester backbone may account for the observations that (A.T)-rich regions of DNA and poly $(\mathrm{dA}-\mathrm{dT})$ differ in several biochemical and physicochemical properties from regions with other sequences. ${ }^{23-27}$ An interpretation of these observations in terms of the alternating DNA model has been given elsewhere. ${ }^{27,28}$

More recent physicochemical evidence regarding the alternating nature of the phosphodiester backbone of poly (dA-dT) comes from $\mathrm{P}^{31}-\mathrm{nmr}$ studies of the highly homogeneous 145 base-pair fragment and a joint $\mathrm{x}$ $\mathrm{ray} / \mathrm{nmr}$ study on fibers of double-helical poly(dA-dT). ${ }^{29,30}$ Alternating structures have been demonstrated for synthetic DNAs in high-salt solution by $n$ mr studies. ${ }^{31}$

\section{Stereochemistry at the Replicating Fork}

The classical autoradiography experiments of Cairns ${ }^{32}$ have shown that DNA duplicates by forming a fork. The two chains of the parent duplex become separated at the fork and pair with newly made complementary chains. Replication is a complex, multistep process, involving a relatively large number of proteins. ${ }^{33}$ 'The crystal conformation observed here may, 
TABLE V

Coordinates $(\AA)$ of the Minihelix in Molecular Inertial System

\begin{tabular}{|c|c|c|c|}
\hline Atom & $x$ & $y$ & $z$ \\
\hline $\mathrm{P} 4$ & 8.595 & -1.385 & -1.278 \\
\hline $\mathrm{O} 1 \mathrm{P} 4$ & 8.414 & -0.097 & -2.065 \\
\hline $\mathrm{O} 2 \mathrm{P} 4$ & 9.787 & -2.216 & -1.418 \\
\hline $\mathrm{O} 3 \mathrm{P} 4$ & 8.44 & -1.169 & 0.239 \\
\hline $\mathrm{O} 5 \mathrm{P} 4$ & 7.357 & -2.241 & -1.609 \\
\hline P3 & 9.221 & 3.713 & 0.59 \\
\hline O1P3 & 8.429 & 4.272 & -0.331 \\
\hline $\mathrm{O} 2 \mathrm{P} 3$ & 10.605 & 3.34 & 0.205 \\
\hline O3P3 & 9.251 & 4.559 & 1.876 \\
\hline O5P3 & 8.408 & 2.504 & 1.056 \\
\hline O1R4 & 4.712 & -3.261 & -0.831 \\
\hline C1R4 & 3.946 & -3.019 & -2.028 \\
\hline C2R4 & 4.677 & -3.126 & -3.2 \\
\hline C3R4 & 5.673 & -4.273 & -2.808 \\
\hline O3R4 & 4.962 & -5.646 & -3.098 \\
\hline C4R4 & 5.731 & -4.055 & -1.288 \\
\hline C5R4 & 7.165 & -3.476 & -1.005 \\
\hline O1R3 & 7.039 & 0.645 & 2.883 \\
\hline C1R3 & 5.782 & 0.007 & 2.578 \\
\hline C2R3 & 6.223 & -0.851 & 1.397 \\
\hline C3R3 & 7.478 & -0.268 & 0.803 \\
\hline C4R3 & 8.085 & 0.332 & 2.126 \\
\hline C5R3 & 9.084 & 1.495 & 1.984 \\
\hline N1T4 & 3.524 & -1.568 & -1.728 \\
\hline $\mathrm{C} 2 \mathrm{~T} 4$ & 2.054 & -1.283 & -1.63 \\
\hline $\mathrm{O} 2 \mathrm{~T} 4$ & 1.273 & -2.234 & -1.665 \\
\hline N3T4 & 1.786 & 0.021 & -1.543 \\
\hline $\mathrm{C} 4 \mathrm{~T} 4$ & 2.638 & 1.085 & -1.539 \\
\hline $\mathrm{O} 4 \mathrm{~T} 4$ & 2.087 & 2.134 & -1.412 \\
\hline C5T4 & 4.018 & 0.721 & -1.635 \\
\hline C6T4 & 4.361 & -0.546 & -1.817 \\
\hline C7T4 & 5.055 & 1.892 & -1.582 \\
\hline N1A3 & 0.857 & 0.535 & 1.655 \\
\hline $\mathrm{C} 2 \mathrm{~A} 3$ & 1.605 & -0.52 & 1.749 \\
\hline N3A3 & 3.010 & -0.519 & 1.993 \\
\hline $\mathrm{C} 4 \mathrm{~A} 3$ & 3.596 & 0.666 & 2.022 \\
\hline C5A3 & 3.03 & 1.894 & 1.804 \\
\hline C6A3 & 1.415 & 1.791 & 1.762 \\
\hline N6A3 & 0.748 & 3.014 & 1.552 \\
\hline N7A3 & 3.880 & 2.984 & 1.932 \\
\hline C8A3 & 4.859 & 2.262 & 2.181 \\
\hline N9A3 & 4.827 & 0.989 & 2.153 \\
\hline $\mathrm{P} 2$ & -8.645 & -1.327 & 1.397 \\
\hline O1P2 & -9.941 & -2.056 & 1.613 \\
\hline $\mathrm{O} 2 \mathrm{P} 2$ & -8.461 & -0.066 & 2.124 \\
\hline $\mathrm{O} 3 \mathrm{P} 2$ & -8.639 & -1.109 & -0.149 \\
\hline $\mathrm{O} 5 \mathrm{P} 2$ & -7.353 & -2.185 & 1.724 \\
\hline $\mathrm{P} 1$ & -8.462 & 3.955 & -0.811 \\
\hline O1P1 & -7.598 & 4.453 & 0.231 \\
\hline $\mathrm{O} 2 \mathrm{P} 1$ & -9.860 & 4.206 & -0.607 \\
\hline O5P1 & -8.142 & 2.447 & $\begin{array}{l}-0.999 \\
\quad(\text { continued })\end{array}$ \\
\hline
\end{tabular}


TABLE V (continued)

\begin{tabular}{lccc}
\hline Atom & $x$ & $y$ & $z$ \\
\hline O3P1 & -7.896 & 4.686 & -2.207 \\
O1R2 & -4.873 & -3.131 & 1.029 \\
C1R2 & -3.984 & -2.65 & 2.155 \\
C2R2 & -4.764 & -2.862 & 3.264 \\
C3R2 & -5.597 & -4.129 & 3.009 \\
O3R2 & -4.887 & -5.319 & 3.31 \\
C4R2 & -5.755 & -4.03 & 1.651 \\
C5R2 & -7.272 & -3.609 & 1.191 \\
O1R1 & -7.181 & 0.552 & -2.926 \\
C1R1 & -5.947 & -0.257 & -2.566 \\
C2R1 & -6.458 & -1.1 & -1.375 \\
C3R1 & -7.681 & -0.341 & -0.826 \\
C4R1 & -8.3 & 0.259 & -1.948 \\
C5R1 & -8.921 & 1.573 & -1.786 \\
N1T2 & -3.587 & -1.342 & 1.796 \\
C2T2 & -2.254 & -1.072 & 1.562 \\
O2T2 & -1.408 & -1.965 & 1.641 \\
N3T2 & -1.910 & 0.228 & 1.393 \\
C4T2 & -2.917 & 1.292 & 1.452 \\
O4T2 & -2.249 & 2.381 & 1.385 \\
C5T2 & -4.177 & 0.918 & 1.563 \\
C6T2 & -4.501 & -0.318 & 1.747 \\
C7T2 & -5.212 & 2.007 & 1.584 \\
N1A1 & -1.026 & 0.368 & -1.682 \\
C2A1 & -1.769 & -0.736 & -1.866 \\
N3A1 & -3.139 & -0.753 & -1.96 \\
C4A1 & -3.557 & 0.496 & -2.091 \\
C5A1 & -3.069 & 1.654 & -2.033 \\
C6A1 & -1.590 & 1.596 & -1.736 \\
N6A1 & -0.875 & 2.608 & -1.645 \\
N7A1 & -3.859 & 2.642 & -2.062 \\
C8A1 & -5.019 & 2.156 & -2.268 \\
N9A1 & -4.952 & 0.775 & -2.306 \\
\hline & & &
\end{tabular}

however, provide a possible model for the spatial relationship between the parent and daughter stems at the fork at a particular stage of the replication process.

The model (shown in Fig. 8) is obtained by extending three minihelices that are adjacent to each other in the crystal structure (enclosed within dotted lines in the schematic diagram). All the stereochemical properties found for the tetranucleotide molecules are retained. $\mathbf{P}$ represents the phosphodiester bridging the parent and daughter duplex, $\omega^{\prime}$ and $\omega$ represent the torsion angles about the in-chain $\mathrm{P}-\mathrm{O}^{\prime}$ and $\mathrm{P}-\mathrm{O}^{\prime}$ bonds. These values are $168^{\circ}$ and $-74^{\circ}$, which are the same as those observed in the crystal structure. They correspond to the trans-gauche $\left(\operatorname{tg}^{-}\right)$conformation for the bridging phosphodiester. This conformation produces an extended structure for the parent DNA chains at the fork unlike the gauche-gauche $\left(g^{-} g^{-}\right)$conformation of the backbone within the helical 

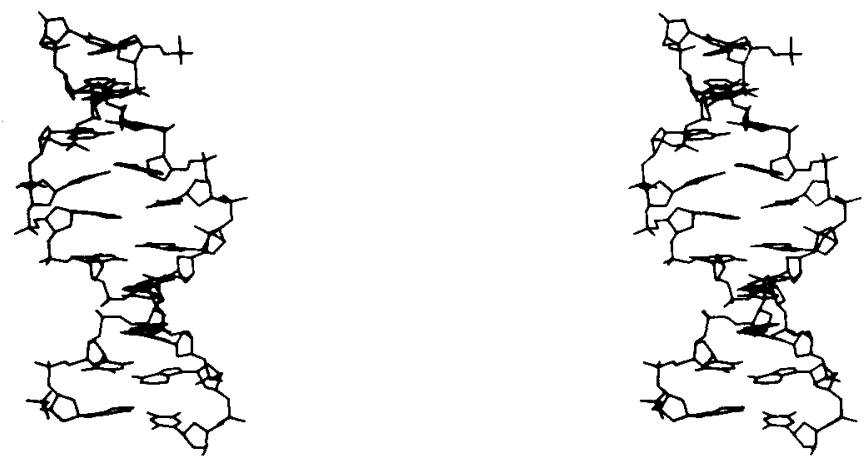

Fig. 7. Computer model of the alternating B-DNA structure obtained by the extension of the minihelix found in the crystal structure.

segments. It also separates adjacent bases on either side of the bridge in each strand by about $14 \AA$, such that the displaced base can pair satisfactorily with a complementary base of the daughter strand. The base pairs adjacent to the phosphodiester bridging $\mathbf{P}$ are at almost the same level, whereas the separation within the duplex region is $3.4 \AA$. The distance between the daughter helix axes is about $23 \AA$, and the angle subtended on the parent helix axis (angle between planes passing through helix axes) is $120^{\circ} \mathrm{C}$. These values are estimated from our model studies, and small changes can be envisaged. It may be noted here that a $\mathrm{tg}^{-}$phosphodiester conformation has been proposed by Sundaralingam et al. ${ }^{34}$ for the messenger RNA during its interaction with the two transfer RNAs.

Several other conformations are possible for the fork depending on the constraints placed on the bridging regions of the backbone. The backbone unit of the polynucleotide chain is composed of six skeletal bonds P-O5',

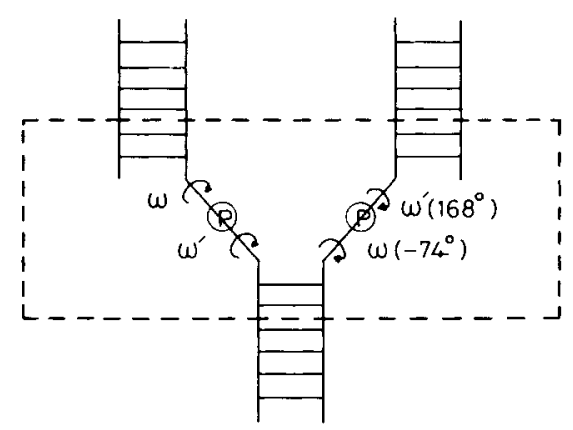

Fig. 8. Stereochemical model of the replication fork, obtained by extending three minihelices found adjacent to each other in the crystal structure (enclosed within dotted line in the schematic diagram). P represents the parent DNA phosphodiester bridging the adjacent nucleotides in the fork region. $\omega^{\prime}$ and $\omega$ are torsions around $\mathrm{P}-\mathrm{O}^{\prime}$ and $\mathrm{P}-\mathrm{O} 5^{\prime}$ bonds, respectively. The backbone conformation is $t g^{-}$and extended at the fork unlike the $g^{-} g^{-}$conformation within the helical limbs. 
$\mathrm{O}^{\prime}-\mathrm{C}^{\prime}, \mathrm{C}^{\prime}-\mathrm{C}^{\prime}, \mathrm{C}^{\prime}-\mathrm{C} 3^{\prime}, \mathrm{C}^{\prime}-\mathrm{O}^{\prime}$, and $\mathrm{O}^{\prime}-\mathrm{P}$. The possibility of rotations about these bonds can result in a large number of spatial relationships among the three DNA limbs at the fork region. Earlier analysis of conformational principles in nucleic acids by Sundaralingam ${ }^{34,35}$ suggests that the flexibility of polynucleotide chains can be regarded mainly in terms of rotations about the bonds linking successive nucleotide units. A large number of conformational possibilities exist even if rotations about the $\mathrm{P}-\mathrm{O}$ linkages alone are considered. The present model shows one such possibility and has the attraction that by a change of one single rotation (about P-O3' of the bridging phosphate) one can bring the three DNA stems at the fork in a satisfactory and close proximity.

We thank the Medical Research Council for financial support and for a Visiting Professorship (to M.A.V.). We are grateful to the D.S.T. and I.C.M.R. (India) for financial support and to SRC for provision of the diffractometer. All crystallographic programs, other than MULTAN, were written by G.M.S. The PLUTO program (unpublished) was provided by Dr. W. D. S. Motherwell.

\section{References}

1. Watson, J. D. \& Crick, F. H. C. (1953) Nature 171, 737.

2. Arnott, S. (1977) in Structure and Properties of Biopolymers, Proceedings of the First Cleveland Symposium on Macromolecules, Walton, A. G., Ed., Elsevier, Amsterdam, 1977.

3. Viswamitra, M. A., Kennard, O., Jones, P. G., Sheldrick, G. M., Salisbury, S., Falvello, L. \& Shakked, Z. (1978) Nature 273,687-688.

4. Wang, A. H.-J., Quingley, G. J., Kolpak, F. J., Crawford, J. L., van Boom, J. H., van der Marel, G. \& Rich, A. (1979) Nature 282, 680-686.

5. Drew, H., Takano, T., Tanaka, S., Itakura, K. \& Dickerson, R. E. (1980) Nature 236, $567-573$.

6. Crawford, J. L., Kolpak, F. J., Wang, A. H.-J., Quigley, G. J., van Boom, J. H., van der Marel, G. \& Rich, A. (1980) Proc. Natl. Acad. Sci. USA 77, 4016-4020.

7. Wing, R., Drew, H., Takano, T., Broka, C., Tanaka, S., Itakura, K. \& Dickerson, R. E. (1980) Nature 287, 755-758.

8. Weimann, G., Schaller, H. \& Khorana, H. G. (1963) J. Am. Chem. Soc. 85, 38353840 .

9. Main, P., Woolfson, M. M., Lessinger, L., Germain, G. \& Declercq, J.-P. (1974) MULTAN, A System of Computer Programs for the Automatic Solution of Crystal Structures from $X$-Ray Diffraction Data, University of York, England.

10. Seeman, N. C., Rosenberg, J. M., Suddath, F. L., Kim, J. J. P. \& Rich, A. (1976) J. Mol. Biol. 104, 109-144.

11. Rosenberg, J. M., Seeman, N. C., Day, R. O. \& Rich, A. (1976) J, Mol. Biol. 104, 145167.

12. Hingerty, B., Subramanian, E., Stellman, S. D., Sato, T., Broyde, S. B. \& Langridge, R. (1976) Acta Crystallogr., Sect. B 32, 2998-3013.

13. Karle, J. (1968) Acta Crystallogr., Sect. B 24, 182-186.

14. Levitt, M. (1978) Proc. Natl. Acad. Sci. USA 75, 640-644.

15. Sundaralingam, M. (1969) Biopolymers 7, 821-860.

16. Viswamitra, M. A., Reddy, B. S., Lin, G. H. \& Sundaralingam, M. (1971) J. Am. Chem. Soc, 93, 4565-4573.

17. Sussman, J. L., Seeman, N. C., Kim, S. H. \& Berman, H. M. (1972) J. Mol. Biol. 66, 403-421.

18. Rubin, J., Brenner, T. \& Sundaralingam, M. (1972) Biochemistry 11, 3112-3228.

19. Camerman, N., Fawcett, J. K. \& Camerman, A. (1976) J. Mol. Biol. 107,601-621. 
20. Motherwell, W. D. S. \& Isaacs, N. W. (1972) J. Mol. Biol. 71, 231-241.

21. Jack, A. (1977) Acta Crystallogr., Sect. A 33, 497-499.

22. Arnott, S., Chandrasekaran, R., Hukins, D. W. L., Smith, P. J. C. \& Watts, L. (1974)

J. Mol. Biol. 88, 523-533.

23. Bram, S. (1971) Nature [New Biol.] 232, 174-176.

24. Johnson, P. H. \& Laskowski, M. S. (1970) J. Biol. Chem. 245, 891-898.

25. Scheffler, I. E., Elson, E. L. \& Baldwin, R. L. (1968) J. Mol. Biol. 36, 291-304.

26. Richmond, T. \& Steitz, T. A. (1976) J. Mol.Biol. 103, 25-38.

27. Viswamitra, M. A., Kennard, O., Shakked, Z., Jones, P. G., Sheldrick, G. M., Salisbury, S. \& Falvello, L. (1978) Curr. Sci. 9, 289-292.

28. Klug, A., Jack, A., Viswamitra, M. A., Kennard, O., Shakked, Z. \& Steitz, T. A. (1979) J. Mol. Biol. 131,669-680.

29. Shindo, H., Simpson, R. T. \& Cohen, J. S. (1979) J. Biol. Chem. 254, 8125-8128.

30. Shindo, H. \& Zimmerman, S. B. (1980) Nature 283, 690-691.

31. Patel, D. J. (1980) in Abstracts of Nucleic Acids: Interactions with Drugs and Carcinogens, British Biophysical Society Meeting, London.

32. Cairns J. (1963) J. Mol. Biol. 6, 208-213.

33. Alberts, B. \& Sternglanz, R. (1977) Nature 269, 655-661, and references cited therein.

34. Sundaralingam, M., Brennan, T., Yathindra, N. \& Ichikawa, T. (1975) in Structure and Conformation of Nucleic Acids and Protein-Nucleic Acid Interaction, Sundaralingam, M. \& Rao, S. T., Eds., University Park Press, Baltimore, pp. 101-113.

35. Sundaralingam, M. (1973) Int. J. Quantum Chem., Quantum Biol. 1, 81-91.

Received April 22, 1981

Accepted July 29, 1981 